Marquette University

e-Publications@Marquette

School of Dentistry Faculty Research and

Publications

Dentistry, School of

$5-1-2018$

\title{
A Hemispherical Contact Model for Simplifying 3D Occlusal Surfaces
}

Miguel Castro-Garcia

University of Castilla-La Mancha

Pedro Ángel Moreno-Cabello

University of Cordoba

Miguel Ángel Rubio-Paramio

University of Jaen

Pilar Carranza-Cañadas

University of Cordoba

Geoffrey A. Thompson

Marquette University, geoffrey.thompson@marquette.edu

Accepted version.The Journal of Prosthetic Dentistry, Vol. 119, No. 5 (May 2018): 804-811. DOI. (C) 2018 Elsevier B.V. Used with permission. 
Marquette University

\section{e-Publications@Marquette}

\section{Dentistry Faculty Research and Publications/School of Dentistry}

This paper is NOT THE PUBLISHED VERSION; but the author's final, peer-reviewed manuscript. The published version may be accessed by following the link in the citation below.

The Journal of Prosthetic Dentistry, Vol. 119, No. 5 (May, 2018): 804-811. DOI. This article is (C) Elsevier and permission has been granted for this version to appear in e-Publications@Marquette. Elsevier does not grant permission for this article to be further copied/distributed or hosted elsewhere without the express permission from Elsevier.

\section{Contents}

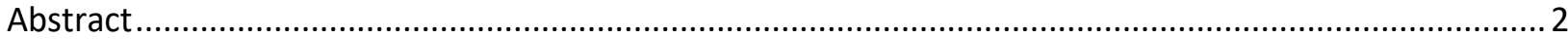

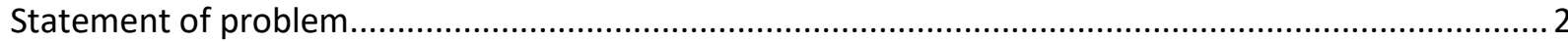

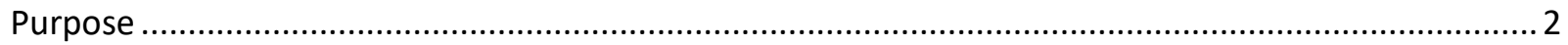

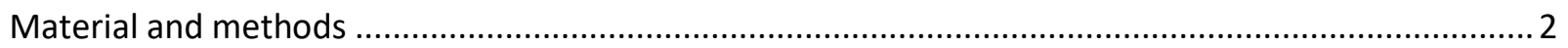

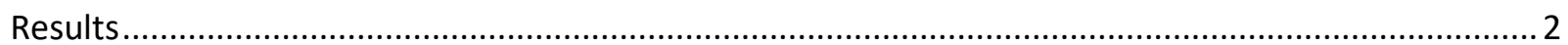

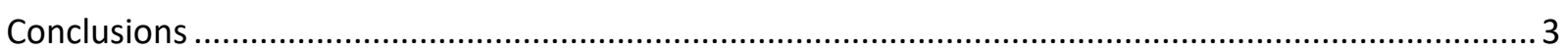

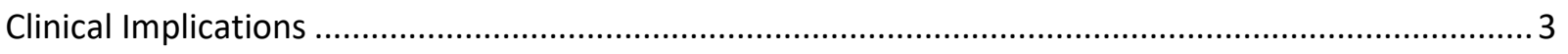

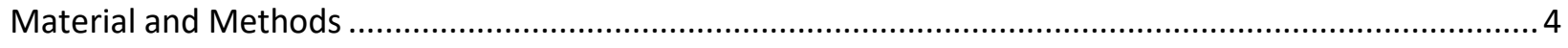

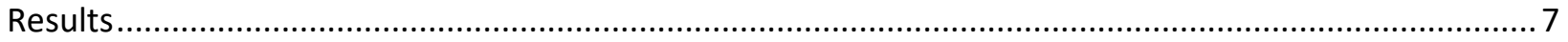

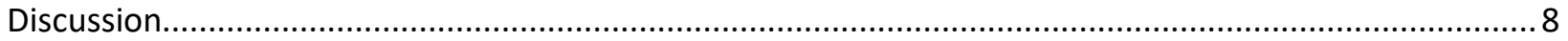

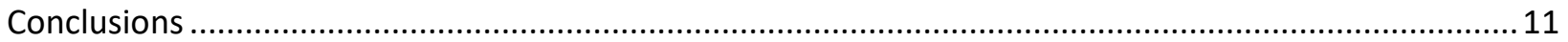

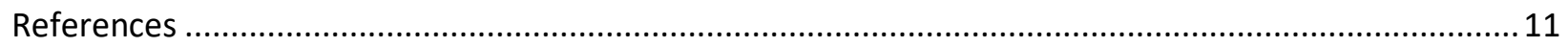

\section{A hemispherical contact model for simplifying 3D occlusal surfaces}




\section{Miguel Castro-Garcia}

Department of Applied Mechanics \& Project Engineering, Industrial Engineering School of Albacete, University of Castilla-La Macha, Spain

\section{Pedro Ángel Moreno-Cabello}

Department of Graphic Engineering \& Geomatics, Biosystems Engineering School, University of Cordoba, Spain

\section{Miguel Ángel Rubio-Paramio}

Department of Graphic Engineering, Design \& Projects, Industrial Engineering School, University of Jaén, Spain

\section{Pilar Carranza-Cañadas}

Department of Graphic Engineering \& Geomatics, Biosystems Engineering School, University of Cordoba, Spain

\section{Geoffrey A. Thompson}

Graduate Prosthodontics, Department of General Dental Sciences, School of Dentistry, Marquette University, Milwaukee, WI

\section{Abstract}

\section{Statement of problem}

Currently, dental articulators can recreate mandibular movements and occlusal contacts. However, whether virtual articulators can also provide information about occluding dental surfaces, functional movements, and the mandibular condyles is unclear.

\section{Purpose}

The purpose of this in vitro study was to evaluate the occluding surfaces on dental casts obtained from a patient and approximate them to a hemispherical contact model. Both models were tested by digitizing the Dentatus ARL dental articulator.

\section{Material and methods}

A combination of photogrammetry and structure from motion methods were used to scan a Dentatus ARL articulator and representative dental casts. Using computer-aided engineering and finite element analysis, contact points and action vectors to the forces on occluding surfaces and condyles were obtained for cast and hemispherical models. This experiment was performed using centric occlusion and 3 different condylar inclinations. The Kruskal-Wallis 1-way analysis_of variance on ranks test was used to allow all pairwise comparisons between condylar inclination and mechanical action vector values in each location $(\alpha=.05)$.

\section{Results}

Action vectors from the cast model and each location of the hemispherical model were calculated to show the mechanical consequences and the similarity among models. Overall, no significant differences were observed for action vectors (A20 versus A40 versus A60) at each location (dental cast/hemisphere, right condylar, and left condylar) in the analysis of dental casts and the hemisphere model

(.382 $\leq P \leq .999)$. 


\section{Conclusions}

This study provided graphical information that may assist the dental professional in determining which occlusal contacts should be modified to attain condylar and balanced centric occlusion.

\section{Clinical Implications}

The hemispherical contact model is a new tool that can assess forces on the dentition and the condyles before, during, or after prosthetic treatment.

Articulators may be used for diagnosis and oral rehabilitation and can simulate mandibular movements and occlusal contacts in the patient's absence. The history of dental articulator design is a testament to the complexities of the craniomandibular system. - However, mandibular movements on different types of articulators often show inconsistent results, and the methodology has been called into question. ${ }^{2,3,4,5}$

Even the most sophisticated devices (mechanical or virtual) for mimicking mandibular movement have deficiencies. $\frac{6}{}$ Nevertheless, virtual articulators may be more advantageous because they can achieve greater accuracy and precision. ${ }^{7,8}$ Virtual articulators also allow the study of mandibular movement at specific times ${ }^{9}$ and consequently enhance the diagnosis, treatment, and confidence in the treatment outcome. Virtual articulators are divided into those based on existing mechanical articulators and those that try to emulate the complex temporomandibular joint (TMJ) system. ${ }^{10}$

Sipilä et al $\underline{11}$ studied the occlusal contacts between opposing dentitions and classified them into 4 categories: protrusion, laterotrusion, mediotrusion, and retrusion interferences. If the curvature from the contacting dental surfaces is analyzed, one curvature predominates over the remainder and can be approximated by a hemisphere. Specifically, the hemisphere represents all possible occlusal contacts between the maxillary and the mandibular teeth. Additionally, the contacting surfaces of each tooth may be fitted to a spherical sector or a portion of the hemisphere. Spherical contacts possess symmetry in all axes, as opposed to the geometric modeling of dental arches proposed by other studies. ${ }^{12,13}$ The resulting action vectors produced by contacts between opposing spherical sectors and obtained by finite element analysis (FEA) depend on the spatial positioning (from the facebow) in the virtual articulator and the digitized patient casts. ${ }^{14}$

The first goal of the present study was to obtain the resultant action vector from the occluding surfaces in centric occlusion and at the condyles by using maxillary and mandibular casts obtained from a dental patient. The second goal was to approximate the patient action vector to a new hemispherical contact model. For this purpose, the model consisted of 2 eccentric hemispheres (the upper hemisphere represents the maxilla and the lower represents the mandible) located spatially at 8 different contact points in the presence of the same experimental boundary conditions as the patient's dental cast. The final consideration was that both experimental models were tested using 3 different condylar inclinations (Cls).

The null hypotheses were that no difference would be found between the magnitude and direction of the action vectors observed in a simulated hemispherical model and the digitized patient model it simulates, and that no difference would be found between the magnitude and direction of the action vectors observed in a simulated hemispherical model and the digitized patient model it simulates as changes in $\mathrm{Cl}$ were made. 


\section{Material and Methods}

This research used a virtual dental articulator based on the Dentatus ARL, designed by Arne G. Lauritzen. It is similar to the Hanau $\mathrm{H} 2$, which has been used in other studies. ${ }^{15,16}$ The study was based on 2 experiments that have in common the use of a virtual Dentatus ARL articulator, but it differed in the models analyzed: the dental cast model and the hemispherical model. The workflow was in 3 stages. The first stage involved acquisition of the 3-dimensional (3D) geometry of the casts of the patient studied and modeling a semiadjustable articulator (Dentatus Articulator ARL; Dentatus) and the hemispherical surfaces in a computer-aided engineering environment. After obtaining institutional review board approval, the dental casts were obtained from a 20 -year-old woman and transferred to the articulator using a Dentatus facebow and jaw relation record. The entire articulator with the articulated casts was then 3D scanned (PhotoScan Professional; Agisoft) with the aim of transferring the data to the virtual environment. The third stage studied the spatial arrangement of the action vectors produced in each of the locations under consideration in response to an applied occlusal load.

The geometry of the patient's casts was obtained by means of photogrammetry techniques and structure from motion. In addition, the geometry of the Dentatus ARL articulator was modeled using computer-aided engineering software (Autodesk Inventor Professional 2015). The various components of the articulator were modeled from a 3D point cloud obtained with photogrammetry and structure from motion to further improve the FEA. Because this type of analysis is quite sensitive to any irregularities, this prevented the introduction of errors caused by small imperfections. ${ }^{17}$

The cleaning and treatment of the point cloud for conversion to a meshed model was accomplished by means of mesh treatment software (MeshLab; Visual Computing Laboratory). As a result, virtual geometries with a maximum absolute global error of $28 \mu \mathrm{m}$ were obtained. This value was within the operating range of different manufacturers (5 to $30 \mu \mathrm{m}) .{ }^{18}$ In addition, this value was less than the tolerances obtained by some digitally printed casts. ${ }^{19}$ Finally, the positions of the maxillary and mandibular casts on the Dentatus ARL articulator were transferred to the virtual articulator using the 3D scanned data.

The hemispherical model used the same virtual articulator modeling, except that the models analyzed were hemispherical models instead of dental casts. With the objective of studying the decomposition of force vectors on the proposed spherical contact, it became necessary to introduce an eccentricity variable (e) (Fig. 1), the presence of which ensured the decomposition of the action vectors in the 3 main spatial directions. The value of e in the tests was $30 \mathrm{~mm}$, while the diameter of the contact area was set to $60 \mathrm{~mm}$. 


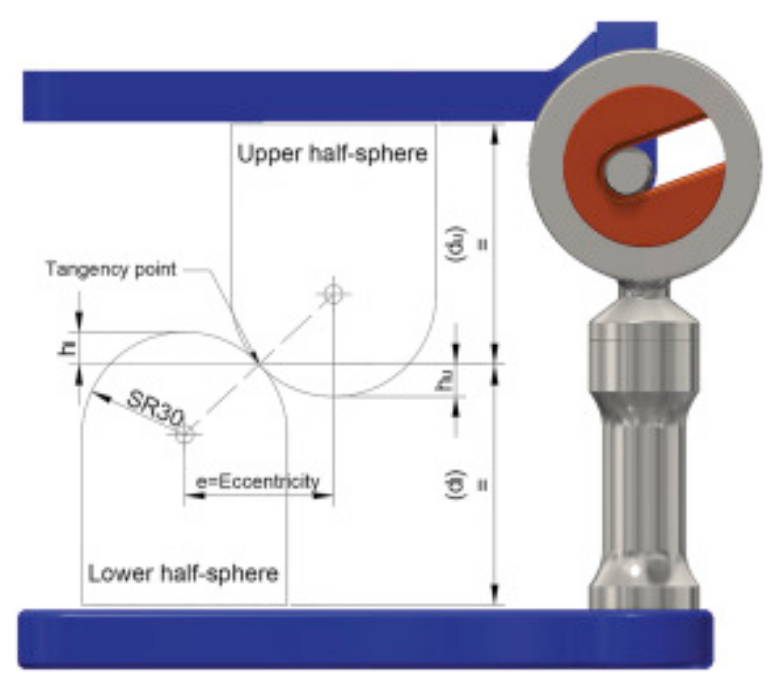

Figure 1. Geometric relationship of hemispherical models at Qd localization.

Another important consideration in the hemispherical model was the location of the tangency point between the hemispherical models. To define this correctly, 4 variables were considered: the height of the upper spherical dome (hu), the height of the lower spherical dome (hl), the distance between the top spherical dome and the upper member of the virtual articulator (du), and the distance between the lower spherical dome and the lower member of the virtual articulator (dl). The experimental setting of these variables was $h u=h l=4 \mathrm{~mm}$ and $d u=d l$. Consequently, the tangency point was always midway between the upper and lower member of the virtual articulator.

In preparation for the analysis of mechanical actions, the mandibular hemispherical model was subdivided into 8 positions equally distributed about the hemisphere and separated by an angle of 45 degrees (Fig. 2). The upper hemispherical model was always fixed at the same place. Clinically, the dental professional could have difficulty in determining the exact dental surface curvature. For this reason, the spherical sector of 45 degrees is proposed as a working unit that is easy to identify by its size.

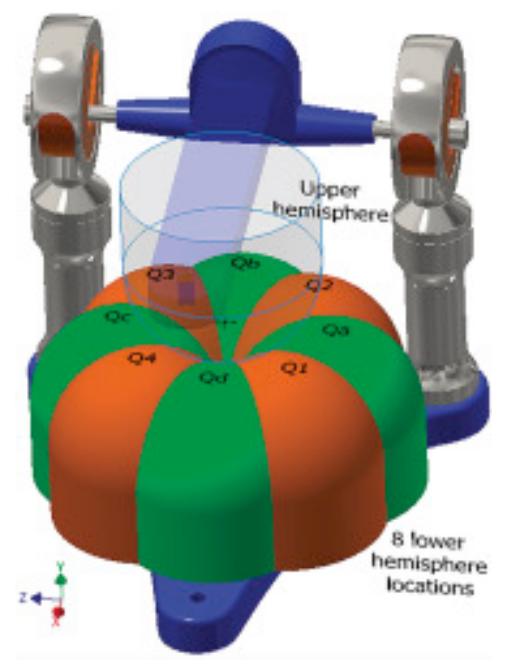

Figure 2. Virtual articulator top view from 8 working positions (Q1 to Q8) 45 degrees from each other in upper hemisphere relative to lower hemisphere. 
The main positions $Q d$ and $Q b$ are in the sagittal plane, and $Q a$ and $Q c$ are in the patient's coronal plane. Intermediate positions (Q1 to Q4) are equidistant to the main positions and separated by an angle of 45 degrees.

The articulator settings are conditional on the type of experiment being performed. In this situation, the movements were similar to protrusive, so the selected Bennett angle value was 0 degrees. To determine if a relationship existed between the $\mathrm{Cl}$ and the mechanical actions analyzed, $3 \mathrm{Cls}$ ( 20 degrees, 40 degrees, and 60 degrees) were selected. A study of responses was made at 8 different positions of the lower hemispherical model. The average $\mathrm{Cl}$ values for men and women are 41.9 degrees with a range of 26.1 to 61.8 degrees (standard deviation 9.2 degrees) ${ }^{20}$

As the boundary conditions for FEA aim to better approximate reality, the materials used for the analysis were dentin and cortical bone. The materials were characterized as isotropic for consistency, although teeth are not strictly isotropic and spherical models show no preferential direction. For the analysis, cortical bone was attributed to the articulator. Regarding the specific mechanical properties, dentin was characterized by a Young modulus of $13.2 \mathrm{GPa}$ and a Poisson ratio of $0.25 .^{21}$ Cortical bone was characterized by a Young modulus of $19.4 \mathrm{GPa}$ and a Poisson ratio at $0.39 .{ }^{22}$

Two loads were introduced into both experimental models. The first was occlusal loading in maximum intercuspation corresponding to a force of $97 \mathrm{~N}$, uniformly distributed on a surface, and approximating $50 \%$ of maximum human occlusal force. ${ }^{23}$ At the same time, the effect of gravity in the vertical axis of the virtual articulator was included.

A tetrahedral mesh was used for discretization of the elements involved in the tests. The average element size (fraction of diameter model) was 0.1 , the minimum element size (fraction of average size) was 0.2 , the grading factor 1.5 , and the maximum turn angle 60 degrees. The analysis was optimized through a convergence of internal tensions, and, by averaging 7 mesh-refinement cycles, the size of the tetrahedron used was independent of the meshed body.

In the presence of all boundary conditions specified, FEA was performed and repeated with 3 different values of $\mathrm{Cl}$ and, in the case of the hemispherical model, at each of the 8 main and intermediate positions of the spherical surfaces. This allowed an objective comparison of the action forces produced by each of the geometric constraints specified above, as shown in Figure 3.

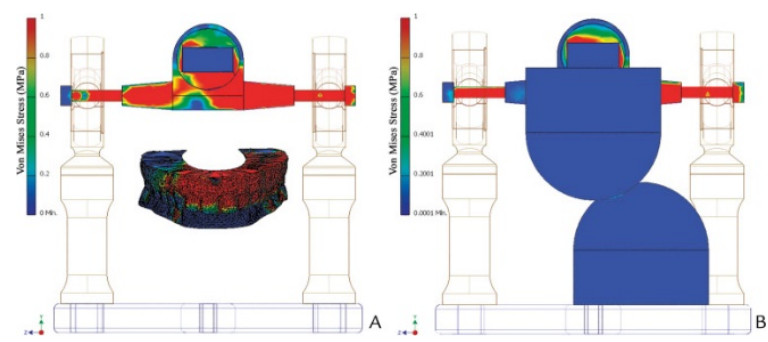

Figure 3. Comparison at condylar inclination of 20 degrees. A, Finite element analysis completed in actual model. B, Hemispherical model at Q2 position. 
The statistical tool used to study this relationship was the Kruskal-Wallis 1-way analysis of variance of ranks, which allowed all-pairwise comparisons between the $\mathrm{Cl}$ and the mechanical action vector values in each location $(\alpha=.05)$.

\section{Results}

The action vector was obtained at each location on the virtual articulator under the boundary conditions specified above - that is, the 3D vector that exerted occlusal loads. ${ }^{24}$ The operation was repeated for each $\mathrm{Cl}$. The resulting vectors for each location (center point from right condyle, center point from left condyle, and centroid from the casts; Fig. 4) were positioned in 3D space. All action vectors had the same global coordinate system and shared the same center point in their respective location. The perspective view of the virtual articulator allowed a comparative analysis of the spatial distribution of the action vectors at each location defined in the actual model (Fig. 4) and the hemispherical models (Fig. 5).

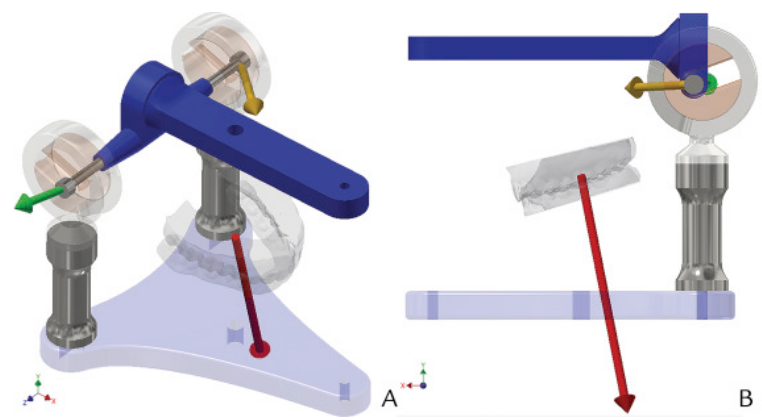

Figure 4. Dental cast model actions at right condyle, left condyle, and center point for condylar inclination of 40 degrees. A, Perspective view. B, Sagittal view.

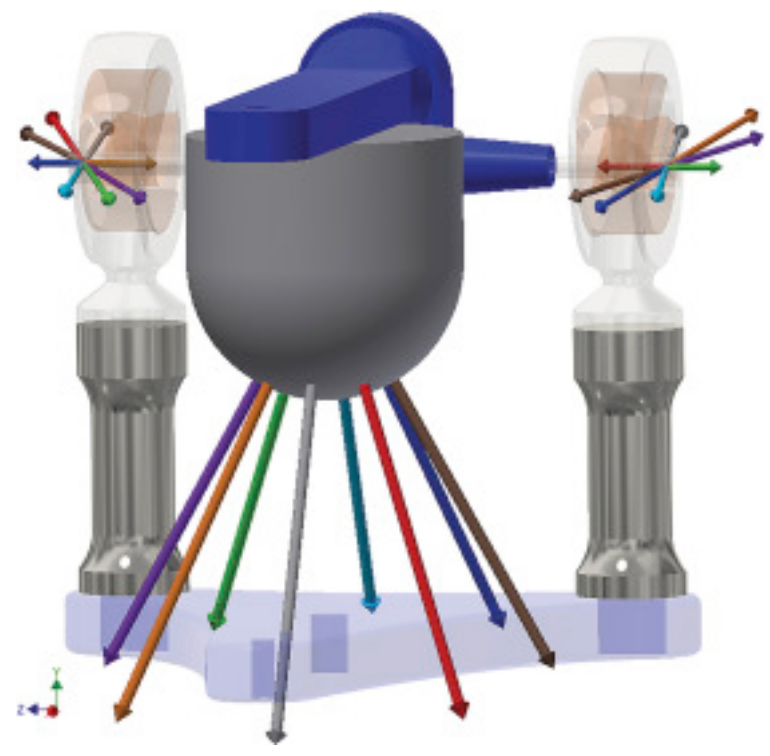

Figure 5. Action vectors between upper and lower hemispherical contacts and condyles for 8 different locations. Condylar inclination 40 degrees.

The action vector was calculated from the FEA data for the $\mathrm{Cl}$ of 20 degrees (A20), 40 degrees (A40), and 60 degrees $(A 60)$ in dental casts $(C)$, in hemisphere $(H)$ models, and at the right condylar $(\mathrm{RC})$ and left 
condylar (LC) positions. The results of the Kruskal-Wallis analyses are presented in Table 1. Overall, no significant differences were observed for action vectors (A20 versus $A 40$ versus $A 60$ ) at each location $(\mathrm{C} / \mathrm{H}, \mathrm{RC}$, or $\mathrm{LC})$ in the analysis of dental casts and hemisphere model $(.382 \leq P \leq .999)$.

Table 1. Probability results of Kruskal-Wallis test for A20 versus A40 versus $A 60$ at each position

\begin{tabular}{|l|l|l|l|l|l|l|l|l|l|}
\hline Location & Casts & Qa & Qb & Qc & Qd & Q1 & Q2 & Q3 & Q4 \\
\hline C/H & 0.933 & 0.829 & 0.993 & 0.993 & 0.993 & 0.929 & 0.999 & 0.993 & 0.993 \\
\hline RC & 0.929 & 0.929 & 0.993 & 0.993 & 0.993 & 0.993 & 0.721 & 0.829 & 0.993 \\
\hline LC & 0.993 & 0.879 & 0.999 & 0.999 & 0.999 & 0.933 & 0.382 & 0.993 & 0.721 \\
\hline
\end{tabular}

$\mathrm{C} / \mathrm{H}$, dental cast/hemisphere; RC, right condylar; LC, left condylar.

\section{Discussion}

The results of this study do not support rejection of the null hypotheses: no differences were found between the magnitude and direction of the action vectors observed in a simulated hemispherical model and the digitized patient model it simulated, and $\mathrm{Cl}$ did not affect the outcomes. No significant differences were found in $A 20$ versus $A 40$ versus $A 60$ for $C, R C$, or $L C$ positions. This means that casts mounted on the articulator in centric occlusion did not vary with changes in $\mathrm{Cl}$. This statement is in accordance with the findings of Christopher et al, ${ }^{25}$ who reported that changes in the location of the condyles had only a small effect on the condylar trajectories of the patient studied. This implies that the character of the action vector will be unique for each patient. Similarly, no significant differences were found in all possible combinations and the $\mathrm{Cl}$ tested for each of the 8 relative hemispherical positions (Table 1). Differences in probability values (Table 1 ) are due to the FEA; the analysis is an approximate method and has slight variance between repetitions.

The location of the action vector depends on the maxillomandibular or hemispherical relationship and is expressed at the condylar and contacting surfaces (Fig. 6).

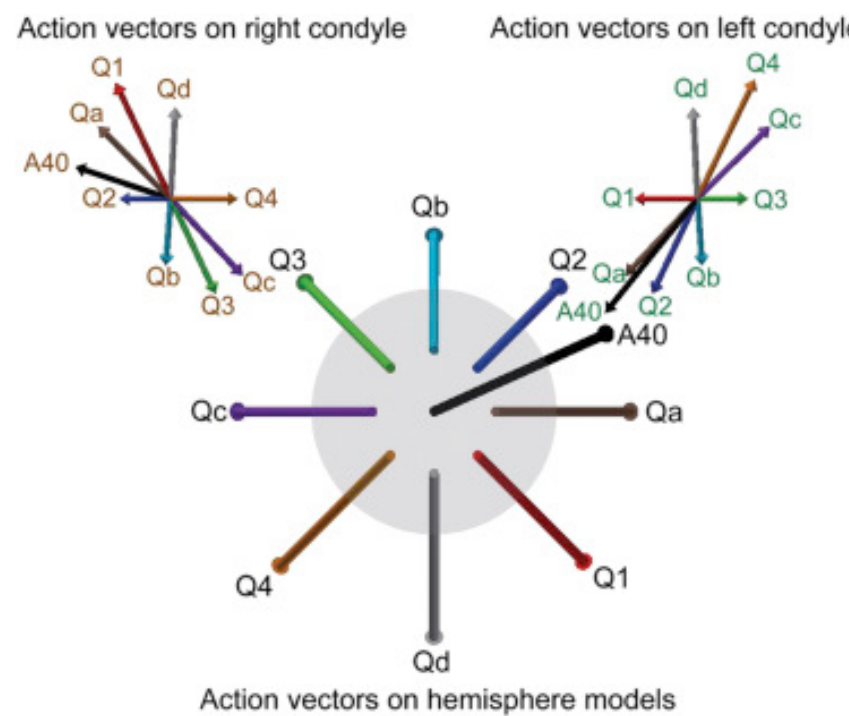

Figure 6. Upper hemispherical model top view (Qi) and cast (A40) action vectors of virtual articulator, where i is 8 relative positions of spheres for condylar inclination of 40 degrees. Each group of action vectors represented by same color. 
Moreover, the authors related the type of contact with the risk of effects in the TMJ or muscles. Accordingly, Figure 7 shows that when the global action vector from the patient or the hemispherical model is in the spherical sector from the arc Q4-Qd-Q1, both condyles exert a compressive force in their glenoid fossae. This scenario can be observed in a retrusive movement. Moreover, this may predict an unstable occlusion and may exhibit structural changes in the TMJ, including bone resorption and fibrocartilage calcification at the condyle because of the transfer of forces at the disk. $\underline{26}$

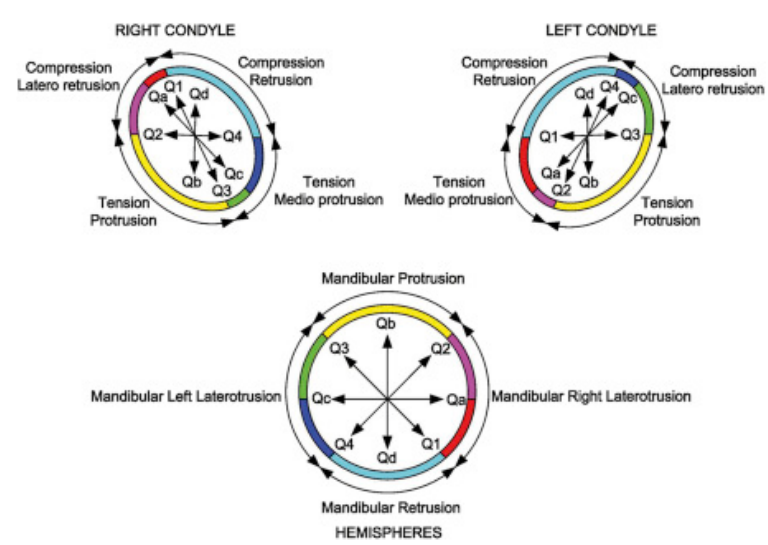

Figure 7. Top view of sectors grouped into colors. Dynamics and mechanical consequences depend on color of sector.

The action vector that belongs to hemisphere locations in the arc Q2-Qb-Q3 means that the mandible is in a protrusive relationship and the masticatory muscles are in a state of tension. Scott and Lundeen ${ }^{27}$ reported that patients who protruded their mandible experienced significantly more pain than control subjects and that this pain was located in the immediate preauricular area. This pain was similar in quality and location to that of many patients diagnosed as having myofascial pain-dysfunction syndrome. Van Boxtel et $\mathrm{al}^{28}$ also commented on the effects of a tension tendency and demonstrated enhanced postural activity in the temporalis muscles in terms of relative contraction strength; this is associated with migraine and muscle contraction headache.

If the action vector from the patient is over the spherical sectors Q1-Qa-Q2, the right condyle produces a laterotrusive (compression) in its glenoid fossa and a mediotrusive (tension) in the left condyle. To help clarify, and regarding the hemisphere positions in Figure 7, the direction of the maxillary action vectors corresponds with the displacement direction in the maxilla. However, the mandibular displacements are in the opposite direction. The complementary scenario happens when the global action vector is over the Q3-Qc-Q4 spherical sector or during a left lateral movement of the mandible. In this case, there is a laterotrusion (compression) on the left condyle, and the right condyle experiences a mediotrusion (tension).

Each laterotrusive scenario may create condylar imbalance in terms of load direction, which has been studied. ${ }^{29}$ For example, Arnett et al ${ }^{30}$ reported that lateral or medial torques may cause condylar resorption and late mandibular relapse. Additionally, Langenbach and Hannam ${ }^{31}$ in their study on muscle simulation reported that the condylar load on the balancing side was less than on the working side. Their finding coincides with the contact hemisphere approximation presented here.

The other observation made is the relative sliding direction between opposing arches (Fig. 7). The hemispherical model demonstrates that when the patient's action vector is over the sectors in the arc 
Q2-Qb-Q3, the displacement of the mandible is anterior. Complementary sectors in Q1-Qd-Q4 are the result of mainly posterior displacement of the mandible. Last, lateral displacement predominates in spherical sectors belonging to arc Q1-Qa-Q2 (right laterotrusion) or Q3-Qc-Q4 (left laterotrusion). In prosthodontic reconstructions, sliding contacts must be controlled. This study can be used to understand sliding surface tendencies and eliminate them.

In the dental cast model, the patient's dental casts tend to slide toward the right; the right condyle exhibits a laterotrusion compression tendency, while the left condyle exhibits mediotrusive tension behavior (Fig. 4). The action vector resulting from the patient's casts is similar to the behavior produced in the hemispherical model between Q2 and Qa, and is labeled A40 in Figure 6. The patient action vector and the hemispherical model have the same lineal system with a lineal response: 2 fixed points (symbolizing the condyles) and 1 contact point (symbolizing the dental cast). Both models possess a uniform geometry in any spatial relationship. The correspondence between the models contributes to the understanding of the human craniomandibular system and could become a tool for classifying occlusal function (Fig. 7) and its effects at the condyle and on the occlusal surfaces (Fig. 8). Figure 8 demonstrates which hemispherical orientation of the occlusal contact tooth surface should receive attention to achieve balance for the patient.

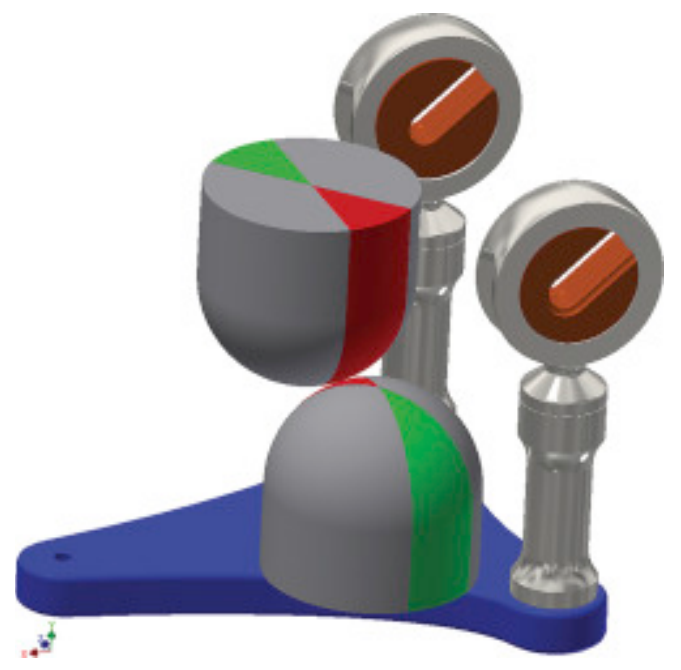

Figure 8. Contact surface between Qa-Q2 locations of upper and lower hemispherical models in red with condylar inclination of 40 degrees; antagonists in green.

The condyles will be in a state of balance when the patient's action vector is in a purely vertical and centered position. The amount of deviation from the vertical and a centered position may be related to the occlusion and subsequently the condylar position. With regard to the studied patient, decomposition of the force vector from the articulated casts resulted in a center point action vector that deviated from the ideal vertical position. To move the center point action vector to its optimum position, it is necessary to increase the contacting surfaces (green sector surfaces)(Fig. 9) or to avoid those corresponding with the red sector surfaces. 


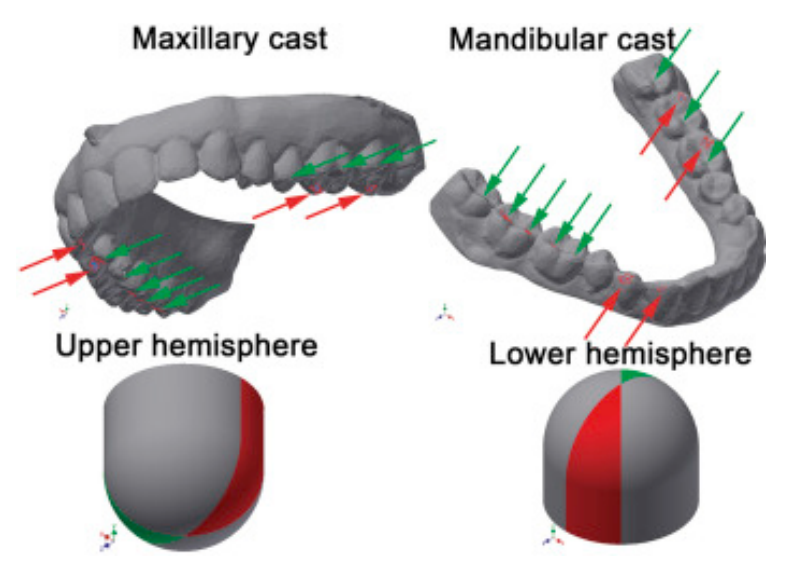

Figure 9. Contact surfaces in maximum occlusion (in red) of casts studied for condylar inclination of 40 degrees, where 2 red areas (removed material) or green area (added material) shown relative to upper and lower hemispheres.

The contacts in each of the patient's casts in the centric occlusion position are shown in Figure 9. If the spherical surface sector (Q2-Qa) as determined by the action vector from the cast model is compared with the geometry of the tooth contacts, it is clear which predominates in the dental system.

In patients with myofascial dysfunction or tooth mobility, the dental surfaces indicated by red arrows in Figure 9 may be treated by removing structure from the surface, keeping in mind the relationship of the cusps and maintenance of the occlusal vertical dimension. Alternatively, adding material in the spherical sectors marked in green will help achieve balance (Fig. 8). This procedure is more appropriately implemented in a prosthetic rehabilitation. Consequently, determining which surfaces contribute to the correction or deviation from a balance of forces is facilitated. However, increasing the number of contacts is not sufficient; rather, it is advisable to know which ones contribute to a slide between opposing surface contacts.

Future research could focus on implementing the hemispherical model on virtual articulators or on studying this new model with regard to the evaluation and health of the craniomandibular system.

\section{Conclusions}

Within the limitations of this in vitro study, the following conclusions were drawn:

1. A unique set of resultant action vectors at the condyles and from the occluding surface of maxillary and mandibular casts in centric occlusion was generated.

2. These action vectors were simulated in a hemispherical model with a high degree of fidelity.

3. The experimental models demonstrated that $\mathrm{Cl}$ had no effect on the magnitude and direction of the patient's action vector.

4. The graphic representation facilitates an understanding of the mechanical behavior of the patient's craniomandibular system.

\section{References}

1 R.L. Engelmeier, D.M. Belles, E.N. Starcke. The history of articulators: the contributions of Rudolph L. Hanau and his company-part I. J Prosthodont, 19 (2010), pp. 409-418 
2 C.C. Beard, K. Donaldson, J.A. Clayton. Comparison of an electronic and a mechanical pantograph. Part I: consistency of an electronic computerized pantograph to record articulator settings. J Prosthet Dent, 55 (1986), pp. 570-574

3 T.M. Chou, C.H. Pameijer. An investigation of the reproducibility of articulators. J Prosthet Dent, 58 (1987), pp. 442-448

4 K. Donaldson, J.A. Clayton. Comparison of mandibular movements recorded by two pantographs. J Prosthet Dent, 55 (1986), pp. 52-58

5 J. Dos Santos Júnior, M.M.J. Ash. A comparison of the equivalence of jaw and articulator movements. J Prosthet Dent, 59 (1988), pp. 36-42

6 J.P. Okeson. Evolution of occlusion and temporomandibular disorder in orthodontics: past, present, and future. Am J Orthod Dentofacial Orthop, 147 (2015), pp. 216-223

7 L. Maestre-Ferrín, J. Romero-Millán, D. Peñarrocha-Oltra, M. Peñarrocha-Diago. Virtual articulator for the analysis of dental occlusion: an update. Med Oral Patol Oral Cir Bucal, 17 (2012), pp. 160163

8 P.R. Koralakunte, M. Aljanakh. The role of virtual articulator in prosthetic and restorative dentistry. J Clin Diagn Res, 8 (2014), pp. 25-28

9 P. Kumar, A. Kumar, A. Khattar, R. Goel. Significance of virtual articulators: an overview. Int J Health Allied Sci (2012), p. 297

$10 \mathrm{~W}$. Wu, Y. Cen, Y. Hong, A. Keeling, B. Khambay. A pilot study to assess the feasibility and accuracy of using haptic technology to occlude digital dental models. J Dent, 46 (2016), pp. 54-60

11 K. Sipilä, P. Zitting, P. Siira, P. Laukkanen, M.R. Järvelin, K.S. Oikarinen, et al. Temporomandibular disorders, occlusion, and neck pain in subjects with facial pain: a case-control study. Cranio, 20 (2002), pp. 158-164

$12 \mathrm{~T}$. Kondo, S.H. Ong, K.W. Foong. Tooth segmentation of dental study models using range images. IEEE Trans Med Imaging, 23 (2004), pp. 350-362

13 D.D. Chung, R. Wolfgramm. Maxillary arch perimeter prediction using Ramanujan's equation for the ellipse. Am J Orthod Dentofacial Orthop, 147 (2015), pp. 235-241

14 L.J. Lux, G.A. Thompson, K.J. Waliszewski, G.J. Ziebert. Comparison of the Kois Dento-Facial Analyzer System with an earbow for mounting a maxillary cast. J Prosthet Dent, 114 (2015), pp. 432-438

$15 \mathrm{M}$. Dora Balos, C. Soaita, D. Cerghizan. A study on the mandibular arc of closure reproducibility using the T-Scan III computerized occlusal analysis system. Procedia Technol, 19 (2015), pp. 916-920

16 E. Solaberrieta, O. Etxaniz, R. Minguez, J. Gorozika, L. Barrenetxea, E. Sierra. Virtual production of dental prostheses using a dental virtual articulator. Int J Interact Des Manuf, 9 (2015), pp. 1930

17 T.J.R. Hughes, J.A. Cottrell, Y. Bazilevs. Isogeometric analysis: CAD, finite elements, NURBS, exact geometry and mesh refinement. Comput Method Appl M, 194 (2005), pp. 39-41

18 A. Ender, A. Mehl. Accuracy of complete-arch dental impressions: a new method of measuring trueness and precision. J Prosthet Dent, 109 (2012), pp. 121-128

19 K.Y. Lee, J.W. Cho, N.Y. Chang, J.M. Chae, K.H. Kang, S.C. Kim, et al. Accuracy of three-dimensional printing for manufacturing replica teeth. Korean J Orthod, 45 (2015), pp. 217-225

20 R.Z. Alshali, R. Yar, C. Barclay, J.D. Satterthwaite. Sagittal condylar angle and gender differences. J Prosthodont, 22 (2013), pp. 561-565 
21 J.H. Kinney, S.J. Marshall, G.W. Marshall. The mechanical properties of human dentin: a critical review and re-evaluation of the dental literature. Crit Rev Oral Biol Med, 14 (2003), pp. 13-29

22 T.M. van Eijden. Biomechanics of the mandible. Crit Rev Oral Biol Med, 11 (2000), pp. 123-136

23 H.J. Schindler, J. Lenz, J.C. Türp, K. Schweizerhof, S. Rues. Small unilateral jaw gap variations: equilibrium changes, co-contractions and joint forces. J Oral Rehabil, 36 (2009), pp. 710-718

24 C.C. Peck. Biomechanics of occlusion-implications for oral rehabilitation. J Oral Rehabil, 43 (2016), pp. 205-214

25 C.P. Christopher, M.M. Greg, W.L.J. Christopher, J.K. Iven. Trajectories of condylar points during nonworking side and protrusive movements of the mandible. J Prosthet Dent, 82 (1999), pp. 322-331

26 M. Bakke, E. Möller. Craniomandibular disorders and masticatory muscle function. Scand J Dent Res, 100 (1992), pp. 32-38

27 D.S. Scott, T.F. Lundeen. Myofascial pain involving the masticatory muscles: an experimental model. Pain, 8 (1980), pp. 207-215

28 A. Van Boxtel, P. Goudswaard, K. Janssen. Absolute and proportional resting EMG levels in muscle contraction and migraine headache patients. Headache, 23 (1983), pp. 215-222

29 B.A. Sutter. Incidence of headaches related to occlusion and bite force imbalance: a case study. Cranio, 34 (2016), pp. 195-207

30 G.W. Arnett, S.B. Milam, L. Gottesman. Progressive mandibular retrusion-idiopathic condylar resorption. Part I. Am J Orthod Dentofacial Orthop, 110 (1996), pp. 8-15

31 G.E. Langenbach, A.G. Hannam. The role of passive muscle tensions in a three-dimensional dynamic model of the human jaw. Arch Oral Biol, 44 (1999), pp. 557-573 